\title{
Paracoccidioidomicosis Diagnóstico por el laboratorio de 333 casos
}

\author{
Castillo J. ${ }^{1}$, Ordóñez N. ${ }^{1}$, López S. ${ }^{2}$, Castañeda E. ${ }^{1}$, Grupo del Programa de Micosis Pulmonares ${ }^{3}$
}

\begin{abstract}
Resumen
La frecuencia con la que se diagnostica la paracoccidioidomicosis en nuestro país sigue apoyando la consideración de que esta entidad constituye la micosis sistémica más importante en nuestro medio. En el presente trabajo se informan 333 casos de pacientes con paracoccidioidomicosis, diagnosticados por los cuatro laboratorios del programa de micosis pulmonares del Instituto Nacional de Salud y por el laboratorio de micología médica del Grupo de Microbiología del mismo instituto, durante un período de 12 años (1981-1992). El diagnóstico fue establecido con la sospecha clínica y una combinación de pruebas de laboratorio con diferentes sensibilidades y especificidades. De los 333 pacientes estudiados, $316(95 \%)$ eran hombres y $17(5 \%)$ mujeres; relación hombre:mujer 18,5:1. El 55,5\% de los pacientes se encontraba en el grupo de edad de 41 a 60 años. La forma clínica predominante fue la forma crónica del adulto $(99 \%)$ con $50,7 \%$ de presentación unifocal. La distribución geográfica fue muy amplia y señaló la importancia de los centros de diagnóstico. Las sensibilidades del $97 \%$ para la inmunodifusión y del $90 \%$ para la fijación del complemento confirmaron su importante valor diagnóstico.
\end{abstract}

\section{Summary}

Paracoccidioidomycosis is the most important systemic mycosis in our country, as demonstrated by the high frequency of diagnosed cases. In this report, 333 paracoccidioidomycosis cases diagnosed at the four laboratories of the Pulmonary Mycoses Program of the Instituto Nacional de Salud and at the Mycology Laboratory of the same institute during a twelve year period are presented. In relation to the age and sex distribution, 316 patients $(95 \%)$ were male, and $17(5 \%)$ female (male:female ratio $18.5: 1$ ) and $55.5 \%$ of the patients were in the age group $41-60$. Regarding the clinical presentation, the chronic adult form was the predominant one $(99 \%)$ with unifocal presentation in $50.7 \%$ of the cases. The ample geographic distribution of the disease demonstrates the importance of the diagnostic centers. The sensitivities of $97 \%$ for immunodiffusion and $90 \%$ for complement fixation validate them as important diagnostic tests.

Grupo de Microbiología, Instituto Nacional de Salud.

Hospital Militar, Santafé de Bogotá.

${ }^{3}$ Ruth de Insignares, Juan Daza, Alvaro Correa. Hospital del Tórax Fernando Troconis, Santa Marta.

Clara Inés de Coppiano, Marta Cecilia Kogson, José Coppiano. Hospital Santa Sofía, Manizales.

Marta Vargas, Pedro Pablo Vásquez. Hospital San Antonio, Villavicencio.

María Claudia Rodríguez. Hospital Erasmo Meoz, Cúcuta. 
La paracoccidioidomicosis constituye la micosis sistémica más importante en nuestro medio por la frecuencia con la que se diagnostica (1-4); es así como desde la primera descripción realizada por Méndez Lemaitre en el año de 1950 (5), se ha incrementado el número de casos publicados en nuestro país (6-12). La distribución geográfica de la paracoccidioidomicosis está limitada a regiones de América del Sur y Central (13-18).

La paracoccidioidomicosis es una entidad crónica que se caracteriza por ser una enfermedad pulmonar primaria a menudo asintomática. A partir del pulmón se disemina y origina úlceras granulomatosas en las mucosas oral y nasal; en algunas ocasiones, la infección se extiende al tejido cutáneo o se vuelve generalizada afectando diferentes órganos de forma sistémica (19-22).

$\mathrm{El}$ agente etiológico es el hongo dimórfico Paracoccidioides brasiliensis, del cual, hasta el momento, sólo se ha determinado suforma asexual de reproducción o anamorfo. No se conoce con certeza la forma ni dónde se encuentra el $P$. brasiliensis en la naturaleza; probablemente la fase de moho o forma infectante del hongo se desarrolla en detritos vegetales en regiones húmedas de las áreas endémicas $(23,24)$. Las aleuroconidias o fragmentos del micelio penetran al huésped por inhalación; pocas horas más tarde, se transforman en levaduras multigemantes, que constituyen la fase parasitaria del hongo y, por tanto, son las que se observan en las muestras clínicas obtenidas de los pacientes $(19,21,25)$.

El diagnóstico de la enfermedad debe sustentarse con las pruebas realizadas en el laboratorio $(21,26)$. Estas incluyen: la visualización del agenteetiológico en las diferentes muestras clínicas, en un examen directo o en biopsias; la recuperación del hongo en el cultivo y las técnicas inmunológicas para determinar la presencia de anticuerpos (26). Estas últimas pruebas se han convertido en una importanteayuda diagnóstica dada su sensibilidad y especificidad (27-28).

En el presente trabajo se informan 333 casos de pacientes con paracoccidioidomicosis, diagnosticados o confirmado el diagnóstico en el laboratorio de micología médica del Grupo de Microbiología del Instituto Nacional de Salud, durante el período comprendido entre los años de 1981 a 1992.

\section{MATERIALES Y METODOS}

Pacientes. Se estudiaron 333 pacientes remitidos al laboratorio de micología del Instituto Nacional de Salud(INS) por diferentes centros hospitalarios del país; 149 pacientes fueron diagnosticados en cuatro laboratorios pertenecientes al Programa de Micosis Pulmonares (PMP); Hospital del Tórax Fernando Troconis en Santa Marta, Magdalena; Hospital San Antonio en Villavicencio, Meta; HospitalSantaSofía en Manizales, Caldas; Hospital Sanatorio Amelia hasta el año de 1989 y, a partir de 1990, el Hospital Erazmo Meoz en Cúcuta, Norte de Santander.

En un formulario se consignaron los datos de edad, sexo, ocupación, procedencia, añoy método de diagnóstico, manifestaciones clínicas y resultados de la radiografía de tórax.

Muestras. De algunos pacientes se obtuvieron muestras de esputos, raspado de lesionescutáneas y mucocutáneas y, en algunos casos, el resultado de la biopsia o autopsia. De todos los pacientes se obtuvo sangre para las pruebas serológicas.

Procesamiento. A partir de los esputos y raspados de las lesiones cutáneas y mucocutáneas, se realizó el examen directo con $\mathrm{KOH}$ al $10 \%$ adicionado de tinta parker azul negra y un cultivo en agar glucosado de Sabouraud adicionado de cloranfenicol y cicloheximida (Mycosel, BBL) y agar extracto de levaduras con los mismos antibióticos. Los medios fueron incubados a $27^{\circ} \mathrm{C}$ por 4 a 6 semanas, las colonias sospechosas de $P$. brasiliensis fueron resembradas enagarinfusión cerebro corazón (BHI, BBL) e incubadas a $37^{\circ} \mathrm{C}$ para demostrar su dimorfismo (29).

\section{Pruebas serológicas}

1. Inmunodifusión en gel de agar (ID). La pruebase realizó inicialmente en agarfenolizado al $1 \%$ (30); a partir del año 1989, el medio empleado fue agarosa al $1 \%$ adicionado de azida de sodio (31). Los antígenos empleados fueron:

1) Paracoccidioidina, antígeno metabólico de la fase de levadura del $P$. brasiliensis preparada por dos métodos: el descrito por Restrepo (30) fue empleado desde 1981 y el descrito por Camargo (31) a partir de 1990. 
2) Histoplasmina, antígeno metabólico de la fase miceliar de H. capsulatum, preparada según el método descrito por Harrel (32).

3) Aspergilinas, antígenos metabólicos de Aspergillus fumigatus, $A$. flavus y $A$. niger preparadas según el método descrito por Palmer (33).

2. Fijación de complemento (FC). Se empleó la técnica del $50 \%$ de hemólisis (34) con tres antígenos: las paracoccidioidinas descritas anteriormente y la histoplasmina y levaduras de $H$. capsulatum (33).

\section{Resultados}

Casos por año. En la tabla 1 se presenta el número de casos diagnosticados por año; se observó un rango entre 12 casos diagnosticados en 1989 y 55 casos diagnosticados en 1984.

Edad y sexo de los pacientes. En la tabla 2 se observa la distribución por sexo y grupos de edad. De los 333 pacientes estudiados, 316 $(95 \%)$ eran hombres y 17 mujeres $(5 \%)$, (relación hombre: mujer 18,5:1). El grupo de edad en el que se encontró el mayor número de pacientes fue el de 51 a 60 años (31\%), seguido por el grupo de 41 a 50 años $(24,3 \%)$. Solamente se diagnosticó la entidad en cuatro hombres jóvenes entre los 11 y los 20 años.

TABLA 1. Número de casos de paracoccidioidomicosis diagnosticados por año.

\begin{tabular}{cc}
\hline AÑO & NUMERO DE CASOS \\
\hline 1981 & 33 \\
1982 & 35 \\
1983 & 36 \\
1984 & 55 \\
1985 & 24 \\
1986 & 19 \\
1987 & 38 \\
1988 & 28 \\
1989 & 12 \\
1990 & 24 \\
1991 & 15 \\
1992 & 14 \\
\hline Total & 333
\end{tabular}

TABLA 2. Distribución de los 333 pacientes con paracoccidioidomicosis por grupos de edad y sexo.

\begin{tabular}{ccc}
\hline GRUPO DE EDAD & $\begin{array}{c}\text { MASCULINO } \\
\mathrm{n}(\%)\end{array}$ & $\begin{array}{c}\text { FEMENINO } \\
\mathrm{n}(\%)\end{array}$ \\
\hline $11-20$ & $4(1,2)$ & 0 \\
$21-30$ & $9(2,7)$ & $1(0,3)$ \\
$31-40$ & $42(12,6)$ & $2(0,6)$ \\
$41-50$ & $79(23,7)$ & $3(0,9)$ \\
$51-60$ & $98(29,4)$ & $5(1,5)$ \\
$61-70$ & $45(13,5)$ & $3(0,9)$ \\
$71-80$ & $6(1,8)$ & $1(0,3)$ \\
SD & $34(10,2)$ & $2(0,6)$ \\
\hline TOTAL & $316(95)$ & $17(5)$ \\
\hline
\end{tabular}

SD: $\sin$ dato

Ocupación. Se obtuvieron datos de273 pacientes; $199(73 \%)$ eran agricultores, $15(5 \%)$ se dedicaban a la construcción, $11(4 \%)$ trabajaban como conductores y, en menor porcentaje, otras actividades como médico, vigilante, estudiante y carnicero.

Distribución geográfica. La figura 1 muestra la distribución geográfica de 273 (82\%) de los 333 casos de paracoccidioidomicosis en los que fue posible determinar su procedencia o lugar de residencia en el momento del diagnóstico; los pacientes fueron remitidos de hospitales y centros de salud de Bogotá, Boyacá, Cesar, Quindío, Huila, Nariño, Santander, Cundinamarca, Guainía y de los cuatro laboratorios del Programa de Micosis Pulmonares.

El mayor número de casos se obtuvo en el departamento del Meta, con 63 pacientes (23\%), seguido por Cundinamarca con $36(13 \%)$ y por Norte de Santander con $27(10 \%)$. Catorce de estos últimos pacientes fueron descritos en una publicacion anterior (10).

Motivo de consulta. Los síntomas observados en 168 de los pacientes (50\%) y que fueron la causa de la consulta médica se observan en la tabla 3. Los síntomas más frecuentes fueron: tos $(72 \%)$, pérdida de peso $(54 \%)$, adinamia $(46 \%)$ y disnea (36\%); otros como fiebre, expectoración, disfonía, adenopatías y cefalea estuvieron presentes en menor proporción. 


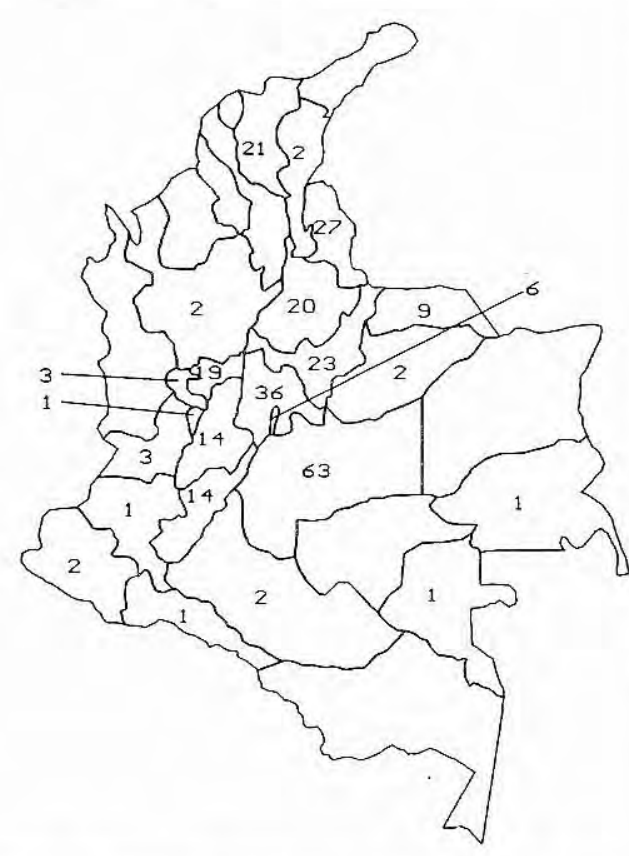

Figura 1. Distribución geográfica de los casos de paracoccidioidomicosis.

TABLA 3. Síntomas observados en 168 pacientes con paracoccidioidomicosis.

\begin{tabular}{lr} 
Tos & $121(72 \%)$ \\
Pérdida de peso & $90(54 \%)$ \\
Adinamia & $78(46 \%)$ \\
Disnea & $61(36 \%)$ \\
Expectoración & $54(32 \%)$ \\
Fiebre & $44(26 \%)$ \\
Astenia & $38(23 \%)$ \\
Hemoptisis & $17(10 \%)$ \\
Disfonía & $12(7 \%)$ \\
Adenopatías & $10(6 \%)$ \\
Cefalea & $8(5 \%)$ \\
Anorexia & $8(5 \%)$ \\
\hline
\end{tabular}

Formas clínicas. Sólo en 298 (89\%) pacientes se obtuvieron datos que permitieron la clasificación de laformaclínica. Trespacientes (1\%) presentaron la forma aguda juvenil y 295 (99\%) presentaron la forma crónica del adulto: $151(50,7 \%)$ con la forma unifocal y $144(48,3 \%)$ la forma multifocal. En esta última el compromiso mucocutáneo fue el más frecuente y se observó en $73(24,5 \%)$ pacientes; otros compromisos están consignadas en la tabla 4.
En 163 pacientes se realizó el diagnóstico diferencial con tuberculosis, estableciéndose las dos entidades en $23(14 \%)$ casos.

Hallazgos radiológicos. Se obtuvo el resultado de la radiografía de tórax en 127 pacientes (tabla 5). La lesión más frecuente fue el infiltrado reticulonodularen $29(23 \%)$, seguido de infiltrado algodonoso en 24 (19\%); se informó fibrosis en 15 pacientes $(12 \%)$, pero sin descripción del tipo encontrado.

Hallazgos del laboratorio: pruebas directas. En 195 pacientes se realizó el examen directo de esputos con $\mathrm{KOH}$ el cual fue positivo en 134 casos $(68,7 \%)$; sólo en 63 de estos casos fue posible realizar el cultivo, recuperándose el hongo en 23 (37\%).

En 49 pacientes que presentaron lesiones cutáneas o mucocutáneas se realizó el examen directo obteniéndose un $100 \%$ de positividad; en 23 de ellos se realizó el cultivo, recuperándose $P$. brasiliensis en 19 (83\%) (tabla 6).

De 84 pacientes se obtuvo el resultado de la biopsia; en 58 (69\%) se informó la presencia de levaduras multigemantes compatibles con $P$. brasiliensis. La cinco autopsias demostraron compromiso micótico de varios órganos como pulmón, hígado, bazo, glándulas suprarrenales, cerebro, riñón y médula ósea.

Hallazgos del laboratorio: pruebas indirectas. De las 333 muestras de sangre procesadas con la técnica de ID, $324(97,3 \%)$ fueron positivas al momento del diagnóstico, determinándose una banda de precipitado en 163 (49\%), dos bandas en $123(37 \%)$ y tres bandas en $38(12 \%)$. Tres pacientes $(0,9 \%)$ presentaron concomitantemente banda $\mathrm{M}$ con la histoplasmina, pero en ninguno se observó banda de precipitado con las aspergilinas. De los nueve pacientes $(2,8 \%)$ negativos en la ID, el diagnóstico se confirmó en tres de ellos por hallazgos en la biopsia, en tres por el examen directo de esputo o lesión positivay en tres por los títulos de fijación de complemento.

La sensibilidad de la ID se determinó tomando como prueba de referencia las pruebas directas: visualización del hongo en las muestras, en las biopsias o en las autopsias o recuperación en el 
TABLA 4. Formas clínicas en 298 pacientes con paracoccidioidomicosis.

\begin{tabular}{lc}
\hline \multicolumn{1}{c}{ FORMA CLINICA } & NUMERO $(\%)$ \\
\hline AGUDA & $3(1)$ \\
CRONICA & $151(50,7)$ \\
UNIFOCAL & \\
MULTIFOCAL & 73 \\
Mucocutánea & 18 \\
Mucocutánea-ganglionar & 21 \\
Mucocutánea-otro órgano* & 5 \\
Ganglionar & 9 \\
Ganglionar-otro órgano* & 9 \\
Cutánea & 4 \\
Cutánea-otro órgano & 5 \\
Otros órganos & \\
\hline Total multifocal & $144(48,3)$ \\
\hline
\end{tabular}

* Hígado, glándula suprarrenal, cerebro, bazo, útero

TABLA 5. Hallazgos radiológicos en 127 pacientes con paracoccidioidomicosis.

\begin{tabular}{lr}
\hline INFILTRADO RETICULONODULAR & $29(23 \%)$ \\
INFILTRADO ALGODONOSO & $24(19 \%)$ \\
INFILTRADO ALVEOLAR PARAHILIAR & $16(13 \%)$ \\
INFILTRADO MICRONODULAR & $16(13 \%)$ \\
FIBROSIS & $15(12 \%)$ \\
INFILTRADO MIXTO & $13(10 \%)$ \\
CAVIDAD & $9(7 \%)$ \\
NODULO & $6(5 \%)$ \\
CALCIFICACIONES & $5(4 \%)$ \\
INFILTRADO PARENQUIMATOSO & $6(5 \%)$ \\
\hline
\end{tabular}

TABLA 6. Resultados del examen directo, cultivo y biopsia en los pacientes con paracoccidioidomicosis.

\begin{tabular}{lrc}
\hline $\begin{array}{c}\text { MUESTRA Y EXAMEN } \\
\text { REALIZADO }\end{array}$ & $n$ & $\begin{array}{c}\text { POSITIVIDAD } \\
n(\%)\end{array}$ \\
\hline $\begin{array}{l}\text { ESPUTOS } \\
\text { Examen directo }\end{array}$ & 195 & $134(68,7)$ \\
Cultivo & 63 & $23(37)$ \\
LESIONES CUTANEAS O & & \\
MUCOCUTANEAS & & \\
EXamen directo & 49 & $49(100)$ \\
Cultivo & 23 & $19(83)$ \\
BIOPSIA & 84 & $58(69)$ \\
AUTOPSIA & 5 & $5(100)$ \\
\hline
\end{tabular}

cultivo. De 188 pacientes con el diagnóstico establecido con las pruebas directas, 183 fueron positivos en la ID, obteniéndose una sensibilidad del $97 \%$.

La cuantificación de los anticuerpos con la técnica de $\mathrm{FC}$ se realizó en 327 pacientes y fue positiva en 281 (85,9\%); ios títulos oscilaron entre 1:8 y mayores de 1:1024, encontrándose el mayor porcentaje $(49,8 \%)$ con títulos entre $1: 32$ y $1: 128$; 18 muestras fueron anticomplementarias.

En la FC se presentaron reacciones cruzadas en 39 de los 281 sueros positivos con los antígenos de H.capsulatum levadura e histoplasmina; en $19(49 \%)$, con los dos antígenos en títulos menores de 1:32; en 18 (46\%), con el antígeno levadura en títulos menores de $1: 64$ y en 2 pacientes $(5 \%)$, con la histoplasmina en títulos menores de 1:16.

La sensibilidad de la prueba se determinó de la misma forma que con la ID; de los 188 pacientes positivos con alguna de las pruebas directas, 168 fueron positivos por la FC, obteniéndose una sensibilidad del $90,3 \%$.

En 152 pacientes pudo hacerse un seguimiento serológico por períodos que oscilaron entre 1 mes hasta 8 años. En 39 pacientes que tuvieron un controi dos meses después del diagnóstico, $23(59 \%)$ reaccionaron de igual forma en 10 frente al antígeno, $14(36 \%)$ disminuyeron el número de bandas y $2(5,1 \%)$ aumentaron el número de bandas.

En el seguimiento empleando la $\mathrm{FC}$, a los dos meses del diagnóstico, 26 pacientes (67\%) conservaron el mismo título, $5(12,8 \%)$ aumentaron en más de dos títulos y $8(20,5 \%)$ disminuyeron en más de 2 diluciones el títuio inicial sin llegar a negativizarse. El mayor porcentaje de negativización ocurrió entre los 13 y los 24 meses, en 10 de 28 pacientes controlados $(36 \%)$.

\section{DISCUSION}

Elnúmero de casos poraño presentó un incremento considerable en 1984, año en el cual los departamentos del Meta y de Norte de Santander ingresaron al Programa de Micosis Pulmonares. Estehailazgo señalala existencia de la enfermedad en la zona y resalta la importancia de instituir este 
tipo de actividad en otros departamentos. El programa tiene como norma el estudio de los pacientes sintomáticos respiratorios, con baciloscopias seriadas negativas, empleando pruebas directas e indirectas para el diagnóstico de micosis pulmonares. De igual manera, la distribución geográfica de los casos diagnosticados puede no corresponder a la distribución real de la enfermedad, sino obedecer a otras causas como son la cercanía y facilidad de algunos hospitales para remitir la muestra a los laboratorios con la infraestructura adecuada; por tanto, en los departamentos en los que no se ha diagnosticado la enfermedad, se puede pensar más en la falta de herramientas de diagnóstico que en la ausencia del agente causal. Adicionalmente, los pocos casos de esta serie provenientes de Antioquia, Valle y Santander, se pueden explicar por el hecho de contar cada uno de esos departamentos con laboratorios de micología, donde se establece el diagnóstico de la entidad en esas zonas $(11,14,15)$.

La cronicidad de la entidad, la no presentación de formas epidémicas, la falta de una prueba intradérmica con sensibilidad y especificidad adecuadas, siguen limitando el estudio de las áreas endémicas para esta micosis en nuestro país (26).

El sexo, la edad y la actividad 'aboral de los pacientes de esta serie están de acuerdo con lo informado en la literatura $(4,12,23,26)$; se pueden resumir como factores de riesgo para sufrir la enfermedad, el ser hombre, la cuarta y sexta década de la vida y estar dedicado a actividades relacionadas con la agricultura. El predominio de la enfermedad en el sexo masculino se ha relacionado recientemente con algunos factores hormonales al demostrarse la inhibición de la transformación del hongo de su fase de moho a la de levadura frente al estradiol, lo que podría ser la causa de la resistencia femenina a sufrir la enfermedad $(28,35)$.

Almomento de la consulta, este grupo de pacientes refería, generalmente, más de un síntoma o signo. El síntoma respiratorio predominante en este grupo fue la tos, en un porcentaje similar al observado por otros autores $(12,14)$; en la mitad de ellos este síntoma se acompañó de expectoración, rara vez hemoptoica.
La forma crónica del adulto fue la forma clínica observada en la mayoría de los pacientes. Dentro de la forma crónica, la unifocal pulmonar fue ligeramente más común que la multifocal; este tipo de distribución se debe, como está claramente establecido, a que la puerta de entrada del hongo es la vía respiratoria, por la inhalación de las aleuroconidias o fragmentos del micelio. Es importante señalar la baja incidencia de la forma mucocutánea (24,5\%); en los informes de Naranjo (36), Londero (20) y Campos (37) la incidencia de este tipo de manifestación fue mayor del $50 \%$. Lo anterior puede obedecer, en parte, al hecho de que el $50 \%$ de los pacientes estudiados provenían de los programas que atienden, en su mayoría, sintomáticos respiratorios.

Laparacoccidioidomicosis juvenil es poco frecuente $y$ tiene un curso agudo y algunas veces fatal. Ochoa (38) informa 4 casos diagnosticados en un período de 13 años, representando el 2,9\% del total de casos estudiados, tal como ocurrió en esta serie.

En nuestro país, donde la tuberculosis es una enfermedad endémica, todo compromiso respiratorio es diagnosticado y tratado como tal, aún en aquellos casos en los cuales las baciloscopias seriadas son negativas. La coexistencia de la paracoccidioidomicosis y la tuberculosis ha sido informada por Gómez (39), en un estudio de 76 pacientes, en el cual $13(17,1 \%)$ presentaban las dos entidades. Spir informa una incidencia concomitante del 10\% (10). En estos casos de concomitancia es difícil determinar cuál de las dos es la enfermedad de base; por tanto, es necesario enfatizar la importancia de hacer el diagnóstico diferencial entre estas dos entidades para que los tratamientos brinden los resultados esperados.

El informe de la radiografía de tórax de los pacientes con paracoccidioidomicosis señala la presencia de infiltrados intersticiales o alveolares, generalmente bilaterales, involucrando la parte media de los pulmones; las cavitaciones son raras perocuando se encuentran son generalmente múltiples (40). Los informes radiológicos de esta serie concuerdan con esta descripción predominando el infiltrado reticulonodular. En otra serie 
colombiana (12), el infiltrado micronodular se encontró en el $71 \%$ de los casos.

El diagnóstico de la paracoccidioidomicosis, como el de otras micosis, debe apoyarse en los recursos que brinda el laboratorio. Debe resaltarse la importancia de realizar un examen directo en fresco, o con $\mathrm{KOH}$, en cualquier tipo de muestra relacionada con el proceso infeccioso, con el fin de visualizar el agente etiológico. En esta serie, el bajo porcentaje de positividad del examen directo comparado con el $90 \%$ informado por otros autores $(10,11)$ pudo deberse al estudio de una sola muestra; se recomienda el análisis de varias muestras, especialmente de esputos, para aumentar la sensibilidad de esta prueba diagnóstica.

Los cultivos presentan menor sensibilidad, la que a su vez depende del tipo de muestra, y requieren de un laboratorio con cierta infraestructura para su realización; la sensibilidad es baja en los casos en que la muestra provenga de sitios en los que se presenta flora bacteriana normal o, cuando en el proceso de obtenerla se contamine con esa flora.

Generalmente, los pacientes con paracoccidioidomicosis presentan una buena respuesta humoral, aunque hasta el momento no se ha podido establecer el verdadero papel de estos anticuerpos (41). De las pruebas serológicas actualmente disponibles para el diagnóstico de la enfermedad, la ID sigue siendo la técnica más utilizada por su sensibilidad y fácil realización, además de su bajo costo. En numerosos trabajos se presenta una sensibilidad mayor del $90 \%$ $(10,11,26,28)$ y en esta serie se confirma este hallazgo. En una baja proporción de los pacientes se determinó la banda $\mathrm{M}$ con la histoplasmina; esta reacción cruzada ha sido informada en la literatura en el mismo bajo porcentaje $(41,42,43)$ y se debe al uso de antígenos crudos.

En los ultimos años, el grupo de Camargo en el Brasil, ha trabajado en la estandarización y purificación de los antígenos para las técnicas serológicas $y$, apoyados por el comité de serodiagnóstico de la Sociedad Internacional de Micología Humana y Animal (ISHAM), extender su empleo en los laboratorios de diagnóstico micológico en varios países de la zona endémica (31). Nuestro laboratorio participó en un estudio patrocinado por ISHAM empleando un antígeno estándar preparado por Camargo. Se analizaron 841 muestras de diferentes países del área endémica de Latinoamérica, observándose un porcentaje de positividad entre el 80 y $91 \%$ (44). Teniendo en cuenta el gran número de sueros probados, los diferentes laboratorios participantes (21 de 5 países) y las variaciones técnicas al realizar la prueba, estos resultados son significativos y permiten seguir trabajando en producir un antígeno único que sea empleado por todos los laboratorios que realicen el serodiagnóstico de esta micosis (44).

Actualmente el grupo de Brasil ha logrado aislar un determinante antigénico específico del $P$. brasiliensis, el cual es una glicoproteína de $43 \mathrm{kD}$ (gp 43) que ha dado buenos resultados en la ID, por lo cual promete convertirse en el antígeno específico para el diagnóstico de esta micosis $(26,31)$.

Una de las primeras pruebas usadas para la detección de anticuerpos anti- $P$. brasiliensis fue la fijación de complemento. A pesar de su buena sensibilidad detectando niveles de anticuerpos tan bajos como 0,1 microgramos $/ \mathrm{ml}$, su uso es limitado por la especificidad. Adicionalmente, la técnica es dispendiosa debido al número de variables que intervienen en ella y que deben ser controladas cada vez que se realiza la prueba (43).

La FC, por su calidad de prueba cuantitativa, tiene valor pronóstico y es adecuada para evaluación de la terapia. La sensibilidad obtenida en esta serie concuerda con la obtenida por otros autores $(11,27,28)$, siendo un poco más baja que la de la ID; a pesar de la baja especificidad, la respuesta generalmente es mayor con el antígeno homólogo pero el número de reacciones cruzadas, especialmente con los antígenos de H. capsulatum, señala la importancia de la interpretación de los resultados (43).

Las dos pruebas serológicas empleadas para el diagnóstico pueden ser útiles para el seguimiento de los pacientes. En cuanto a la ID, la reducción en la intensidad o en el número de bandas 
(cuando se emplea el antígeno de Restrepo) indica una respuesta satisfactoria al tratamiento; igualmente, la reducción en los títulos de FC (43). Sin embargo, los pocos datos referentes al tratamiento en esta serie no permiten profundizar sobre este aspecto ni sacar conclusiones generales; por tanto, su valor pronóstico debe mirarse individualmente.

\section{Referencias}

1. Restrepo A, Robledo M, Giraldo R, et al. The gamut of paracoccidioidomycosis. Am J Med 1976; 61:35.

2. Giraldo R, Restrepo A, Gutiérrez F, et al. Pathogenesis of paracoccidiodomycosis: a model based on the study of 46 patients Mycopathologia 1976; 58:63.

3. Restrepo A, Gutiérrez F, Sanclemente M, Castañeda E, Calle G. Paracoccidioidomycosis (South American Blastomycosis). A study of 39 cases observed in Medellín Colombia. Am J Trop Med Hyg 1970; 19:68.

4. Castañeda E, Ordónez N, Bustos L, Sánchez J. Paracoccidioidomicosis. Diagnóstico por el laboratorio de 87 casos. Acta Med Col 1981; 6:339.

5. Méndez Lemaitre A. Blastomicosis suramericana y otras micosis en Colombia. Rev Hosp Samaritana 1950; 1:3.

6. Retamoso B. Blastomicosis suramericana. Revista Sociedad Med-quirúrgica del Atlántico 1965; 9:91.

7. Díaz D, Gómez G. Granuloma por paracoccidioidomicosis del cerebelo. Neurología Col 1984; 8:150.

8. Lobo CA. Paracoccidiomicosis, presentación de 14 casos. Acta Med Col 1987; 12: 386.

9. Rincón M. Paracoccidioidomicosis. Informe de 138 casos diagnosticados en Santander. UIS Salud 1989; 17:51.

10. Spir N. Paracoccidioidomicosis. Revisión retrospectiva de 100 pacientes. Medicina CES 1991; 5:125.

11. Villar LA, Gómez A, Rincón M, Olarte E, Bárcenas C, Silva F. Paracoccidioidomicosis en Santander: aspectos clínico-epidemiológicos (1953-1992). Médicas UIS 1992; 6:182.

12. Robledo MA, Arango M, Restrepo A. Polimorfismo de las lesiones cutáneas en la paracoccidioidomicosis. Revista de la Sociedad Colombiana de Dermatología 1992; 2:5.

13. Greer D, Restrepo A. La epidemiología de la paracoccidioidomicosis. Bol Oficina Sanit Panam 1977; 428.

14. Rolón PA. Paracoccidioidomicosis, epidemiología en la República del Paraguay, centro de Sur América. Mycopathologia 1976; 59:67.

15. Londero AF, Ramos CD, LópezJ. Progressive pulmonary paracoccidioidomicosis. A study of 34 cases observed in Rio Grande do Sul (Brazil). Mycopathologia 1978;63:53.

16. Asconeguy FR, Bonasse J, Calegari LF, Conti Díaz IA. Paracoccidioidomicosis. A propósito de tres nuevos casos nacionales. Mycopathologia 1982 78:155.
17. Hernández-Pérez E, Orellano Díaz O. Paracoccidioidomicosis. Report of the first autochtonous case in El Salvador. Int J Derm 1984; 23:617.

18. Arauz JC, Mattera RJ, Lucentini MO, Del Prado C. Paracoccidioidomicosis, recopilación de 18 casos. Medicina (Buenos Aires) 1987; 47:337.

19. Lacaz C, Porto E, Costa Martins JE. Micología médica: fungos, actinomicetos e algas de interesse médico. Octava edición, Sao Paulo: editora Sarvier 1991; 248.

20. Londero AT, Ramos CD. Paracoccidioidomicose. Estudio clinico e micologico de 260 casos observados no interior do Estado do Rio Grande do Sul. J Pneumol 1990; 16:129.

21. Franco M, Poncio Méndez R, Moscardi-Bacchi $M$, Rezkallah-Iwasso $M$, Rubens $M$. Paracoccidioidomycosis. Bailliere's Clin Trop Med Commun Dis 1989; $4: 185$.

22. Biagioni L, Souza MJ, Chamma LG, Méndez RP, Marquez AS, Mota NGS, Franco M. Serology of paracoccidioidomycosis. II.Correlation between classspecific antibodies and clinical forms of the disease. Trans Royal Soc Trop Med Hyg 1984; 78:617.

23. Restrepo A. The ecology of Paracoccidioides brasiliensis: a puzzle still unsolved. J Med Vet Mycol 1985; 23:323.

24. Albornoz MB. Isolation of Paracoccidioides brasiliensis from rural soil in Venezuela. Sabouraudia 1971; 9:248.

25. McEwen JG, Bedoya V, Patiño MM, Salazar ME, Restrepo A. Experimental murine paracoccidioidomycosis induced by the inhalation of conidia. J Med Vet Mycol 1987; 25:165.

26. Brummer E, Castañeda E, Restrepo A. Paracoccidioidomycosis: an update. Clin Microbiol Rev 1993; 6:89.

27. Cano LE, Restrepo A. Predictive value of serologic test in the diagnosis and follow of patients with paracoccidioidomycosis. Rev Inst Med Sao Paulo 1987; 29:276.

28. Del Negro G, García N, Rodríguez E, Caria MI, Aguilar M, Lirio V, Lacaz C. The sensitivity, specificity and efficiency values of some serological test in the diagnosis of paracoccidioidomycosis. Rev Inst Med Trop Sao Paulo 1991; 33:277.

29. Ordóñez N, Castillo J, Castañeda E. Micologia Médica. En Microbiología Médica. Manual de Procedimientos. Serie de publicaciones Cientificas, \#14. Bogotá: Instituto Nacional de Salud. 1988; 201.

30. Palmer D, Kaufman L, Kaplan W, Cavallaro JJ. Serodiagnosis of mycotic diseases. Primera edición Springfield, Illinois: Charles C. Thomas Publisher 1977; 118.

31. Camargo ZP, Unterkirchen C, Campoy S, Travassos L.Production of Paracoccidioides brasiliensis exoantigens for immunodifussion tests. J Clin Microbiol 1988; 26(10):2147.

32. Restrepo A. La prueba de la inmunodifusión en el diagnóstico de la paracoccidioidomicosis. Sabouraudia $1966 ; 4: 223$. 
33. Harrell W, Ashworth H, Britt L, George J, Gray S, Green J, Gross H, Johnson J. Specification and evaluation methods for immunological and microbiological reagents. Vol 1: Bacterial, Fungal, Parasitic. Atlanta, Georgia Department of Health, Education, and Welfare: Public Health Service 1976; 107.

34. Palmer DF, Casey H. A guide to the performance of the standardized diagnostic complement fixation method and adaptation to microtest. Atlanta, Georgia U.S. Department of Health, Education and Welfare; Public Health Service 1974; 1.

35. Restrepo A, Salazar ME, Cano LE, Stover E, Feldman D, Stevens DA. Estrogens inhibit mycelium- to-yeast transformation in the fungus Paracoccidiodes brasiliensis: implications for resistence of females to paracoccidioidomycosis. Infect Immun 1984; 46:346.

36. Naranjo MS, Trujillo M, Múnera MI, Restrepo P, Gómez I, Restrepo A. Treatment of paracoccidioidomycosis with itraconazole. J Med Vet Mycol 1991; 28:67.

37. Campos EV, Sartori JC, Hetch ML, Franco MF. Clinical and serological features of 47 patients with paracoccidioidomycosis treated by amphotericin B. Rev Inst Med trop Sao Paulo 1984; 26:179.
38. Ochoa MT, Franco L, Restrepo A. Características de la paracoccidioidomicosis infantil: informe de cuatro casos. Medicina UPB 1991; 10:97.

39. Gómezl. Asociación paracoccidioidomicosis-tuberculosis. Estudio en trece sasos. Ant Med 1979; 28:63.

40. Londero AT, Del Negro G. Paracoccidioidomicose. I. Patogenia, formas clínicas, manifestaciones pulmonares e diagnostico. II. Tratamiento: nocoes prácticas. JPneumol 1986; $12: 41$.

41. Kaufman L. Evaluation of serological test for paracoccidioidomycosis. Procedings 1st Panamerican Symposium on Paracoccidioidomycosis. PAHO Scientific Publication 1972; 221.

42. Restrepo A, Moncada LH. Characterization of the precipitin bands detected in the immunodiffusion tests for paracoccidioidomycosis. Appl Microbiol 1974;28:138.

43. Restrepo A. Procedimientos serológicos en la paracoccidioidomicosis. Adel Microbiol Enf Infecc 1984; 3:182.

44. Restrepo A. Report of activities of the committee on paracoccidioidomycosis serodiagnosis. Mycoses ISHAM Newsletter 1992; 59:4. 